\title{
Fast model-fitting of Bayesian variable selection regression using the iterative complex factorization algorithm (Supplementary)
}

Quan Zhou and Yongtao Guan

\section{S1 Numerical comparison studies of the iterative methods using simulated datasets}

In Section 2.4, we performed numerical comparison studies using real GWAS datasets, where each covariate follows a binomial distribution. For comparison, we conducted the same experiment using simulated datasets where $X_{i j}$ is continuous variable. Let $X_{(i)}$ be an arbitrary row of $\boldsymbol{X}$. First, we sampled $X_{(i)}$ from a multivariate normal distribution with fixed pairwise correlation $r$; that is, $X_{(i)}$ is an independent sample from $\operatorname{MVN}\left(0, \boldsymbol{C}_{r}\right)$ where $\left(\boldsymbol{C}_{r}\right)_{i j}=1$ if $i=j$ and $\left(\boldsymbol{C}_{r}\right)_{i j}=r$ otherwise. Second, we sampled $X_{(i)}$ from a multivariate log-normal distribution such that $\log X_{(i)} \sim \operatorname{MVN}\left(0, \boldsymbol{C}_{r}\right)$. For both cases, we tried $r=0,0.2,0.5,0.9$. Note that for the log-normal case, the pairwise correlation is then equal to $0,0.13,0.38,0.85$, respectively. Then we applied the same procedure and parameter values described in Section $2.4(n=3000$ and $\left.\sigma_{\beta}=0.5\right)$ and collected the wall time usage of the iterative methods for solving 1,000 independent linear systems with form (11). The only difference is that we used the relaxation parameter $\omega_{\mathrm{SOR}}=0.4$ for the successive over-relaxation method since it appeared to produce best overall performance. The results are summarized in Table S1. In almost every scenario, ICF exhibits an overwhelming advantage, especially when the data is heavy-tailed (the log-normal case). The only exception is the normal data with $r=0.9$, where CG also works well. Compared with the results given in Section 2.4, the advantage of ICF over the Cholesky decomposition becomes more prominent. Lastly, we note that GS and SOR work poorly when the pairwise correlation $r \geq 0.2$, and as $r$ grows larger, they quickly become ineffective. This phenomenon will be discussed in the next section.

\section{S2 Numerical studies on the convergence rates of the iterative methods}

\section{S2.1 Symmetric Toeplitz systems}

In this section, we assume the covariance matrix of $\boldsymbol{X}$ is a symmetric Toeplitz matrix, which we will define shortly, denoted by $\boldsymbol{T}$. Instead of sampling $\boldsymbol{X}$, we simply let $\boldsymbol{X}^{t} \boldsymbol{X}=n \boldsymbol{T}$ and consider the linear system

$$
\boldsymbol{A} \hat{\boldsymbol{\beta}}=\left(n \boldsymbol{T}+\sigma_{\beta}^{-2} \boldsymbol{I}\right) \hat{\boldsymbol{\beta}}=\boldsymbol{z} .
$$

We will use several typical choices of $\boldsymbol{T}$ to study how the convergence rates of the iterative methods change with $p$ and $\sigma_{\beta}$. As shown by Proposition 1 , the convergence rate of ICF is given by the spectral radius of $\boldsymbol{\Psi}(\omega)$. For SOR, using the notation introduced in (12), the convergence rate is the spectral radius of $(\boldsymbol{D}+\omega \boldsymbol{L})^{-1}[(1-\omega) \boldsymbol{D}-\omega \boldsymbol{U}]$, which we denote by $\rho_{\mathrm{SOR}}(\omega)$. By letting $\omega=1$, we get the convergence rate of GS, which is $\rho_{\mathrm{GS}}=\rho\left((\boldsymbol{D}+\boldsymbol{L})^{-1} \boldsymbol{U}\right)$. Let $\lambda_{\max }$ and $\lambda_{\min }$ be the largest and smallest eigenvalue of $\boldsymbol{A}$ and thus the condition number of $\boldsymbol{A}$ is $\kappa(\boldsymbol{A})=\lambda_{\max } / \lambda_{\min }$. Then the convergence rate of CG can be bounded from above by $\bar{\rho}_{\mathrm{CG}}=(\sqrt{\kappa(\boldsymbol{A})}-1) /(\sqrt{\kappa(\boldsymbol{A})}+1)$. Note that we cannot compute the exact convergence rate 


\begin{tabular}{|c|c|c|c|c|c|c|c|c|c|c|}
\hline \multirow{2}{*}{ Dataset } & \multirow{2}{*}{$p$} & \multicolumn{5}{|c|}{ Time (in seconds) } & \multicolumn{4}{|c|}{ Convergence failures } \\
\hline & & Chol & $\mathrm{ICF}$ & GS & SOR & CG & $\mathrm{ICF}$ & GS & SOR & $\mathrm{CG}$ \\
\hline \multirow{5}{*}{$\begin{array}{c}\text { Normal } \\
r=0\end{array}$} & 50 & 0.031 & 0.017 & 0.017 & 0.063 & 0.023 & 0 & 0 & 0 & 0 \\
\hline & 100 & 0.19 & 0.06 & 0.06 & 0.25 & 0.09 & 0 & 0 & 0 & 0 \\
\hline & 200 & 1.35 & 0.25 & 0.29 & 1.08 & 0.38 & 0 & 0 & 0 & 0 \\
\hline & 500 & 20.6 & 1.8 & 2.7 & 9.7 & 3.2 & 0 & 0 & 0 & 0 \\
\hline & 1000 & 159 & 7.3 & 21 & 72 & 21 & 0 & 0 & 0 & 0 \\
\hline \multirow{5}{*}{$\begin{array}{l}\text { Normal } \\
r=0.2\end{array}$} & 50 & 0.032 & 0.017 & 0.180 & 0.089 & 0.025 & 0 & 0 & 0 & 0 \\
\hline & 100 & 0.19 & 0.06 & 1.82 & 0.61 & 0.10 & 0 & 525 & 0 & 0 \\
\hline & 200 & 1.36 & 0.25 & 7.21 & 6.19 & 0.38 & 0 & 1000 & 31 & 0 \\
\hline & 500 & 20.6 & 1.8 & 44.6 & 44.5 & 3.6 & 0 & 1000 & 1000 & 0 \\
\hline & 1000 & 159 & 7.7 & 205 & 204 & 23 & 0 & 1000 & 1000 & 0 \\
\hline \multirow{5}{*}{$\begin{array}{l}\text { Normal } \\
r=0.5\end{array}$} & 50 & 0.032 & 0.018 & 0.527 & 0.368 & 0.026 & 0 & 1000 & 0 & 0 \\
\hline & 100 & 0.19 & 0.07 & 1.90 & 1.91 & 0.09 & 0 & 1000 & 1000 & 0 \\
\hline & 200 & 1.35 & 0.26 & 7.21 & 7.22 & 0.46 & 0 & 1000 & 1000 & 0 \\
\hline & 500 & 20.6 & 2.0 & 44.6 & 44.5 & 3.2 & 0 & 1000 & 1000 & 0 \\
\hline & 1000 & 159 & 10 & 195 & 195 & 20 & 0 & 1000 & 1000 & 0 \\
\hline \multirow{5}{*}{$\begin{array}{l}\text { Normal } \\
r=0.9\end{array}$} & 50 & 0.033 & 0.027 & 0.527 & 0.530 & 0.027 & 0 & 1000 & 1000 & 0 \\
\hline & 100 & 0.19 & 0.10 & 1.90 & 1.91 & 0.10 & 0 & 1000 & 1000 & 0 \\
\hline & 200 & 1.35 & 0.42 & 7.21 & 7.22 & 0.40 & 0 & 1000 & 1000 & 0 \\
\hline & 500 & 20.7 & 3.0 & 44.6 & 44.5 & 3.5 & 0 & 1000 & 1000 & 0 \\
\hline & 1000 & 159 & 12 & 190 & 189 & 18 & 0 & 1000 & 1000 & 0 \\
\hline \multirow{5}{*}{$\begin{array}{c}\text { Log-normal } \\
r=0\end{array}$} & 50 & 0.032 & 0.011 & 0.015 & 0.058 & 0.026 & 0 & 0 & 0 & 0 \\
\hline & 100 & 0.19 & 0.04 & 0.06 & 0.23 & 0.10 & 0 & 0 & 0 & 0 \\
\hline & 200 & 1.35 & 0.17 & 0.28 & 1.00 & 0.43 & 0 & 0 & 0 & 0 \\
\hline & 500 & 20.6 & 1.7 & 2.5 & 8.9 & 3.5 & 0 & 0 & 0 & 0 \\
\hline & 1000 & 159 & 7.4 & 21 & 69 & 23 & 0 & 0 & 0 & 0 \\
\hline \multirow{5}{*}{$\begin{array}{c}\text { Log-normal } \\
r=0.2\end{array}$} & 50 & 0.032 & 0.016 & 0.091 & 0.072 & 0.030 & 0 & 0 & 0 & 0 \\
\hline & 100 & 0.19 & 0.06 & 0.84 & 0.34 & 0.12 & 0 & 0 & 0 & 0 \\
\hline & 200 & 1.37 & 0.25 & 7.18 & 2.84 & 0.56 & 0 & 958 & 0 & 0 \\
\hline & 500 & 20.7 & 1.8 & 44.5 & 44.4 & 4.9 & 0 & 1000 & 980 & 0 \\
\hline & 1000 & 159 & 7.6 & 205 & 205 & 37 & 0 & 1000 & 1000 & 0 \\
\hline \multirow{5}{*}{$\begin{array}{l}\text { Log-normal } \\
r=0.5\end{array}$} & 50 & 0.033 & 0.018 & 0.519 & 0.196 & 0.034 & 0 & 815 & 0 & 0 \\
\hline & 100 & 0.19 & 0.06 & 1.90 & 1.80 & 0.17 & 0 & 1000 & 494 & 0 \\
\hline & 200 & 1.36 & 0.25 & 7.21 & 7.22 & 0.76 & 0 & 1000 & 1000 & 0 \\
\hline & 500 & 20.5 & 1.8 & 44.6 & 44.5 & 7.4 & 0 & 1000 & 1000 & 0 \\
\hline & 1000 & 159 & 7.5 & 195 & 194 & 56 & 0 & 1000 & 1000 & 0 \\
\hline \multirow{5}{*}{$\begin{array}{c}\text { Log-normal } \\
r=0.9\end{array}$} & 50 & 0.032 & 0.018 & 0.527 & 0.530 & 0.044 & 0 & 1000 & 1000 & 0 \\
\hline & 100 & 0.19 & 0.07 & 1.90 & 1.91 & 0.19 & 0 & 1000 & 1000 & 0 \\
\hline & 200 & 1.35 & 0.32 & 7.22 & 7.23 & 0.96 & 0 & 1000 & 1000 & 0 \\
\hline & 500 & 20.5 & 2.4 & 44.6 & 44.5 & 9.3 & 0 & 1000 & 1000 & 0 \\
\hline & 1000 & 159 & 13 & 203 & 202 & 74 & 0 & 1000 & 1000 & 0 \\
\hline
\end{tabular}

Table S1: Wall time usage (in seconds) and numbers of convergence failures with simulated data. For normal data, $r$ is the correlation between any two different covariates; for log-normal data, it is the correlation between the logarithms of two different covariates. The statistics for each of the five methods were obtained from 1,000 independent repeats. Chol: Cholesky decomposition; ICF: iterative complex factorization; GS: Gauss-Seidel method; SOR: successive over-relaxation; CG: conjugate gradient. "Convergence failures" columns give the numbers of experiments that fail to converge within 200 iterations for the four iterative methods. 
of $\mathrm{CG}$, and in fact, $\mathrm{CG}$ can be regarded as a direct method since it always converges within $p$ iterations (Trefethen and Bau III, 1997, Lec. 38).

We denote a symmetric Toeplitz matrix by $\boldsymbol{T}\left(a_{0}, \ldots, a_{p-1}\right)$, which satisfies $T_{i j}=a_{|i-j|}$, i.e.

$$
\boldsymbol{T}\left(a_{0}, \ldots, a_{p-1}\right)=\left[\begin{array}{cccccc}
a_{0} & a_{1} & a_{2} & \cdots & \cdots & a_{p-1} \\
a_{1} & a_{0} & a_{1} & \ddots & & a_{p-2} \\
a_{2} & a_{1} & \ddots & \ddots & \ddots & \vdots \\
\vdots & \ddots & \ddots & \ddots & a_{1} & a_{2} \\
a_{p-2} & & \ddots & a_{1} & a_{0} & a_{1} \\
a_{p-1} & \cdots & \cdots & a_{2} & a_{1} & a_{0}
\end{array}\right] .
$$

We consider two structured choices of $\boldsymbol{T}$.

(i) $\boldsymbol{T}_{c}(r)=\boldsymbol{T}(1,-r, r,-r, r, \ldots)$. For a regression problem, this is equivalent to $\boldsymbol{T}(1, r, r, \ldots, r)$ up to sign flipping of the regression coefficients.

(ii) $\boldsymbol{T}_{e}(\ell)=\boldsymbol{T}\left(a_{0}, \ldots, a_{p-1}\right)$ where $a_{k}=\exp (-k / \ell)$ and $\ell>0$. This can be seen as the covariance matrix of a discretized Ornstein-Uhlenbeck process.

Note that in this setup, since

$$
\boldsymbol{A}=n \boldsymbol{T}+\sigma_{\beta}^{-2} \boldsymbol{I}=n\left(\boldsymbol{T}+\frac{1}{n \sigma_{\beta}^{2}} \boldsymbol{I}\right),
$$

the convergence rates of the iterative methods only depend on $\boldsymbol{T}$ and the product $n \sigma_{\beta}^{2}$. Hence we can simply let $n$ be an arbitrary positive constant, and we used $n=1000$. However, for a real scatter matrix $\boldsymbol{X}^{t} \boldsymbol{X}$, one should be careful when $n<p$ since $\boldsymbol{X}^{t} \boldsymbol{X}$ is then rank deficient. Such ill-conditioned systems will be studied in Section S2.2.

We first fixed $\sigma_{\beta}=0.5$ and studied how the convergence rates change with $p$ using five covariance matrices: $\boldsymbol{T}_{c}(0.01), \boldsymbol{T}_{c}(0.1), \boldsymbol{T}_{c}(0.5), \boldsymbol{T}_{e}(5), \boldsymbol{T}_{e}(50)$. The results are summarized in Table S2. As expected, as $p$ grows larger, the convergence rate becomes slower for every method. For the covariance matrix $\boldsymbol{T}_{e}$, since $T_{i j}$ decreases exponentially as $|i-j|$ increases, the impact of larger values of $p$ is not significant. Strikingly, ICF has a much faster convergence rate than all the other methods, even if we simply choose $\omega=1$. In contrast, the convergence rates of GS and SOR quickly approach 1 as $p$ increases. This is probably because GS, SOR (and also Jacobi iteration) favor diagonally dominant matrices for $\boldsymbol{A}$. Hence, if for each row of $\boldsymbol{A}$, the sum of off-diagonal entries increases linearly with $p$, these methods would easily run into convergence difficulties.

Next, we fixed $p=200$ and studied how the convergence rates change with $\sigma_{\beta}$ using $\boldsymbol{T}_{c}(0.1)$ and $\boldsymbol{T}_{e}(5)$. The results are summarized in Table S3. As expected, a smaller value of $\sigma_{\beta}$ makes $\boldsymbol{A}$ diagonally more dominant, and as a result, GS and SOR converge faster. However, ICF exhibits an opposite trend: as $\sigma_{\beta}$ increases, ICF converges faster. This is probably due to the use of the Cholesky decomposition of $\boldsymbol{X}^{t} \boldsymbol{X}$, which implies that if $\sigma_{\beta} \uparrow \infty$, ICF would converge immediately. Note that, because it is the product $n \sigma_{\beta}^{2}$ that really matters, if $p$ and $\sigma_{\beta}$ are fixed and $n$ increases, ICF will converge faster while the other methods become slower. 


\begin{tabular}{|c|c|c|c|c|c|c|c|c|c|}
\hline $\begin{array}{c}\text { Covariance } \\
\text { matrix }\end{array}$ & $p$ & $\sqrt{\kappa(\boldsymbol{A})}$ & $\omega_{\mathrm{ICF}}^{\star}$ & $\omega_{\mathrm{SOR}}^{\star}$ & $\rho(\boldsymbol{\Psi}(1))$ & $\rho\left(\boldsymbol{\Psi}\left(\omega^{\star}\right)\right)$ & $\rho_{\mathrm{GS}}$ & $\rho_{\mathrm{SOR}}$ & $\bar{\rho}_{\mathrm{CG}}$ \\
\hline \multirow{5}{*}{$\boldsymbol{T}_{c}(0.01)$} & 50 & 1.23 & 0.9999 & 0.95 & $2.3 \mathrm{e}-04$ & $1.2 \mathrm{e}-04$ & 0.104 & 0.080 & 0.102 \\
\hline & 100 & 1.42 & 0.9997 & 0.90 & $6.0 \mathrm{e}-04$ & $3.0 \mathrm{e}-04$ & 0.214 & 0.171 & 0.172 \\
\hline & 200 & 1.74 & 0.9993 & 0.81 & 0.0013 & $6.5 \mathrm{e}-04$ & 0.395 & 0.321 & 0.269 \\
\hline & 500 & 2.46 & 0.999 & 0.61 & 0.0027 & 0.0014 & 0.700 & 0.576 & 0.421 \\
\hline & 1000 & 3.33 & 0.998 & 0.42 & 0.0040 & 0.0020 & 0.875 & 0.745 & 0.538 \\
\hline \multirow{5}{*}{$\boldsymbol{T}_{c}(0.1)$} & 50 & 2.56 & 0.998 & 0.63 & 0.0032 & 0.0016 & 0.710 & 0.602 & 0.438 \\
\hline & 100 & 3.47 & 0.998 & 0.42 & 0.0046 & 0.0023 & 0.883 & 0.764 & 0.553 \\
\hline & 200 & 4.81 & 0.997 & 0.26 & 0.0060 & 0.0030 & 0.963 & 0.871 & 0.656 \\
\hline & 500 & 7.50 & 0.996 & 0.12 & 0.0078 & 0.0039 & 0.993 & 0.945 & 0.765 \\
\hline & 1000 & 10.6 & 0.996 & 0.06 & 0.0089 & 0.0044 & 0.998 & 0.972 & 0.827 \\
\hline \multirow{5}{*}{$\boldsymbol{T}_{c}(0.5)$} & 50 & 7.11 & 0.993 & 0.22 & 0.013 & 0.0066 & 0.985 & 0.939 & 0.753 \\
\hline & 100 & 10.0 & 0.992 & 0.12 & 0.016 & 0.0077 & 0.996 & 0.969 & 0.818 \\
\hline & 200 & 14.1 & 0.991 & 0.06 & 0.017 & 0.0087 & 0.9990 & 0.984 & 0.868 \\
\hline & 500 & 22.3 & 0.990 & 0.03 & 0.020 & 0.0097 & 0.9998 & 0.994 & 0.914 \\
\hline & 1000 & 31.5 & 0.990 & 0.01 & 0.021 & 0.010 & 0.99996 & 0.997 & 0.938 \\
\hline \multirow{5}{*}{$\boldsymbol{T}_{e}(5)$} & 50 & 9.51 & 0.984 & 1.06 & 0.032 & 0.016 & 0.747 & 0.719 & 0.810 \\
\hline & 100 & 9.74 & 0.984 & 1.07 & 0.032 & 0.016 & 0.772 & 0.748 & 0.814 \\
\hline & 200 & 9.81 & 0.984 & 1.08 & 0.032 & 0.016 & 0.792 & 0.774 & 0.815 \\
\hline & 500 & 9.84 & 0.984 & 1.05 & 0.032 & 0.016 & 0.804 & 0.797 & 0.815 \\
\hline & 1000 & 9.84 & 0.984 & 1.02 & 0.032 & 0.016 & 0.809 & 0.806 & 0.815 \\
\hline \multirow{5}{*}{$\boldsymbol{T}_{e}(50)$} & 50 & 51.4 & 0.838 & 1.00 & 0.387 & 0.162 & 0.965 & 0.965 & 0.962 \\
\hline & 100 & 64.1 & 0.837 & 1.06 & 0.388 & 0.163 & 0.970 & 0.970 & 0.969 \\
\hline & 200 & 74.4 & 0.837 & 1.00 & 0.389 & 0.163 & 0.973 & 0.973 & 0.973 \\
\hline & 500 & 81.7 & 0.837 & 0.95 & 0.389 & 0.163 & 0.976 & 0.975 & 0.976 \\
\hline & 1000 & 83.7 & 0.837 & 0.94 & 0.389 & 0.163 & 0.977 & 0.976 & 0.976 \\
\hline
\end{tabular}

Table S2: Relationship between $p$ and the convergence rates of iterative methods for solving Toeplitz systems $\boldsymbol{A} \hat{\boldsymbol{\beta}}=\boldsymbol{z}$ where $\boldsymbol{A}=n\left(\boldsymbol{T}+\left(n \sigma_{\beta}^{2}\right)^{-1} \boldsymbol{I}\right)$. See the main text for the structure of $\boldsymbol{T}$. For all the experiments, we used $n \sigma_{\beta}^{2}=250 . \kappa(\boldsymbol{A})$ is the condition number of $\boldsymbol{A} . \omega_{\mathrm{ICF}}^{\star}$ and $\omega_{\mathrm{SOR}}^{\star}$ refer to the optimal relaxation parameters for ICF and SOR respectively. $\rho$ means convergence rate, i.e. the spectral radius of the iteration matrix. An iterative method is convergent if $\rho<1$. In particular, $\rho(\boldsymbol{\Psi}(1))$ refers to ICF with $\omega=1 ; \rho\left(\Psi\left(\omega^{\star}\right)\right)$ refers to ICF with $\omega=\omega_{\mathrm{ICF}}^{\star} ; \rho_{\mathrm{SOR}}$ refers to SOR with $\omega=\omega_{\mathrm{SOR}}^{\star} ; \bar{\rho}_{\mathrm{CG}}$ is the upper bound of the convergence rate of CG. 


\begin{tabular}{clllllllll}
\hline $\begin{array}{c}\text { Covariance } \\
\text { matrix }\end{array}$ & $\sigma_{\beta}$ & $\sqrt{\kappa(\boldsymbol{A})}$ & $\omega_{\mathrm{ICF}}^{\star}$ & $\omega_{\mathrm{SOR}}^{\star}$ & $\rho(\boldsymbol{\Psi}(1))$ & $\rho\left(\boldsymbol{\Psi}\left(\omega^{\star}\right)\right)$ & $\rho_{\mathrm{GS}}$ & $\rho_{\mathrm{SOR}}$ & $\bar{\rho}_{\mathrm{CG}}$ \\
\hline & 10 & 4.82 & 0.999992 & 0.25 & $1.5 \mathrm{e}-05$ & $7.5 \mathrm{e}-06$ & 0.963 & 0.871 & 0.656 \\
& 1 & 4.82 & 0.9992 & 0.25 & 0.0015 & $7.5 \mathrm{e}-04$ & 0.963 & 0.871 & 0.656 \\
$\boldsymbol{T}_{c}(0.1)$ & 0.5 & 4.81 & 0.997 & 0.26 & 0.0060 & 0.0030 & 0.963 & 0.871 & 0.656 \\
& 0.2 & 4.76 & 0.982 & 0.26 & 0.038 & 0.018 & 0.961 & 0.868 & 0.653 \\
& 0.1 & 4.58 & 0.931 & 0.27 & 0.148 & 0.069 & 0.956 & 0.858 & 0.642 \\
& 0.05 & 4.05 & 0.788 & 0.33 & 0.539 & 0.212 & 0.932 & 0.821 & 0.604 \\
\hline & 10 & 10.0 & 0.99996 & 1.07 & $8.1 \mathrm{e}-05$ & $4.1 \mathrm{e}-05$ & 0.799 & 0.779 & 0.818 \\
$\boldsymbol{T}_{e}(5)$ & 1 & 9.96 & 0.996 & 1.08 & 0.0081 & 0.0040 & 0.798 & 0.777 & 0.817 \\
& 0.5 & 9.81 & 0.984 & 1.08 & 0.032 & 0.016 & 0.793 & 0.774 & 0.815 \\
& 0.2 & 8.96 & 0.912 & 1.05 & 0.193 & 0.088 & 0.761 & 0.755 & 0.799 \\
& 0.05 & 4.10 & 0.749 & 0.99 & 0.672 & 0.251 & 0.698 & 0.697 & 0.753 \\
& 4.56 & 0.547 & 0.87 & 1.66 & 0.453 & 0.600 & 0.556 & 0.640 \\
\hline
\end{tabular}

Table S3: Relationship between $\sigma_{\beta}$ and the convergence rates of iterative methods for solving Toeplitz systems $\boldsymbol{A} \hat{\boldsymbol{\beta}}=\boldsymbol{z}$ where $\boldsymbol{A}=n\left(\boldsymbol{T}+\left(n \sigma_{\beta}^{2}\right)^{-1} \boldsymbol{I}\right)$. For all the experiments, we used $n=1000$ and $p=200$. See Table S2 for the annotations of column headers.

\section{S2.2 III-conditioned systems}

Our last numerical study concerned ill-conditioned linear systems. Let $\boldsymbol{C}$ be the covariance matrix of $\boldsymbol{X}$ and consider the linear system $\boldsymbol{A} \hat{\boldsymbol{\beta}}=\boldsymbol{z}$ where

$$
\boldsymbol{A}=n \boldsymbol{C}+\sigma_{\beta}^{-2} \boldsymbol{I}=n\left(\boldsymbol{C}+\frac{1}{n \sigma_{\beta}^{2}} \boldsymbol{I}\right) .
$$

The matrix $\boldsymbol{A}$ is always full rank due to the shrinkage/regularization term $\sigma_{\beta}^{-2} \boldsymbol{I}$; however, it can be ill-conditioned if $\boldsymbol{C}$ is ill-conditioned and $\sigma_{\beta}$ is large.

We still fixed $n=1000, p=200$ and tried different values for $\sigma_{\beta}$. For $\boldsymbol{C}$, we considered the following three choices so that it is ill-conditioned: Toeplitz matrices $\boldsymbol{T}_{c}(0.95)$ and $\boldsymbol{T}_{e}(500)$ (see the last section for definition) and the matrix $\tilde{\boldsymbol{T}}_{c}(20,0.9)$ defined by

$$
\left[\tilde{\boldsymbol{T}}_{c}(m, r)\right]_{i j}=\left\{\begin{array}{cc}
1, & \text { if } i=j, \\
1, & \text { if } i=2 k-1, j=2 k, k=1, \ldots, m, \\
1, & \text { if } i=2 k, j=2 k-1, k=1, \ldots, m, \\
r, & \text { otherwise }
\end{array}\right.
$$

That is, $\tilde{\boldsymbol{T}}_{c}(20,0.9)$ represents the scenario where there are 20 duplicate pairs of covariates (1 and 2,3 and $4, \ldots, 39$ and 40 ), and all the remaining pairs have correlation equal to 0.9 . Hence, the matrix $\tilde{\boldsymbol{T}}_{c}(20,0.9)$ has rank equal to $p-20$. The results are summarized in Table S4. ICF is still much better than all the other methods except when the covariance matrix is $\boldsymbol{T}_{e}(500)$ and $\sigma_{\beta} \leq 0.2$. For a given covariance matrix, ICF favors larger values of $\sigma_{\beta}$ while the other methods favor small values of $\sigma_{\beta}$. Hence, we can also conclude that for an ill-conditioned covariance matrix and a fixed $\sigma_{\beta}$, ICF converges faster for larger sample sizes, which is a very appealing property since the other methods usually fail in such cases. Lastly, for the rank-deficient covariance matrix $\tilde{\boldsymbol{T}}_{c}(20,0.9)$, the optimal relaxation parameter for ICF appears to be around $2 / 3$ and the corresponding convergence rate is about $1 / 3$. Interestingly, we tried $\tilde{\boldsymbol{T}}_{c}(m, r)$ with other values for $m$ and $r$, and made the same observation. 


\begin{tabular}{clllllllll}
\hline $\begin{array}{c}\text { Covariance } \\
\text { matrix }\end{array}$ & $\sigma_{\beta}$ & $\sqrt{\kappa(\boldsymbol{A})}$ & $\omega_{\mathrm{ICF}}^{\star}$ & $\omega_{\mathrm{SOR}}^{\star}$ & $\rho(\boldsymbol{\Psi}(1))$ & $\rho\left(\boldsymbol{\Psi}\left(\omega^{\star}\right)\right)$ & $\rho_{\mathrm{GS}}$ & $\rho_{\mathrm{SOR}}$ & $\bar{\rho}_{\mathrm{CG}}$ \\
\hline & 10 & 61.6 & 0.9997 & 0.03 & $5.2 \mathrm{e}-04$ & $2.6 \mathrm{e}-04$ & 0.99997 & 0.9992 & 0.968 \\
& 1 & 61.0 & 0.974 & 0.03 & 0.053 & 0.026 & 0.99997 & 0.9992 & 0.968 \\
$\boldsymbol{T}_{c}(0.95)$ & 0.5 & 59.3 & 0.900 & 0.03 & 0.221 & 0.100 & 0.99997 & 0.9991 & 0.967 \\
& 0.2 & 50.3 & 0.518 & 0.03 & 1.86 & 0.482 & 0.99996 & 0.999 & 0.961 \\
& 0.1 & 35.6 & 0.132 & 0.04 & 13.2 & 0.868 & 0.99991 & 0.998 & 0.945 \\
\hline & 10 & 417.4 & 0.995 & 1.05 & 0.0100 & 0.0050 & 0.998 & 0.998 & 0.995 \\
$\boldsymbol{T}_{e}(500)$ & 1 & 296.6 & 0.668 & 0.92 & 0.995 & 0.332 & 0.997 & 0.997 & 0.993 \\
& 0.5 & 187.6 & 0.336 & 0.71 & 3.95 & 0.664 & 0.996 & 0.995 & 0.989 \\
& 0.2 & 82.3 & 0.080 & 0.42 & 23.0 & 0.920 & 0.996 & 0.987 & 0.976 \\
& 10 & 41.7 & 0.030 & 0.27 & 65.4 & 0.970 & 0.996 & 0.972 & 0.953 \\
\hline$\tilde{\boldsymbol{T}}_{c}(20,0.9)$ & 0.5 & 212.2 & 0.660 & 0.17 & 1.03 & 0.340 & 0.99993 & 0.9993 & 0.991 \\
& 0.2 & 84.9 & 0.651 & 0.08 & 1.07 & 0.349 & 0.99991 & 0.998 & 0.977 \\
& 0.1 & 42.5 & 0.635 & 0.04 & 1.15 & 0.365 & 0.9999 & 0.996 & 0.954 \\
\hline
\end{tabular}

Table S4: Relationship between $\sigma_{\beta}$ and the convergence rates of iterative methods for solving ill-conditioned systems. For all the experiments, we used $n=1000$ and $p=200$. See Table S2 for the annotations of column headers.

\begin{tabular}{|c|c|c|c|c|c|c|c|}
\hline \multirow{2}{*}{$n$} & \multirow{2}{*}{$p$} & \multicolumn{3}{|c|}{ IND } & \multicolumn{3}{|c|}{ DEP } \\
\hline & & $\operatorname{mean}\left(\eta_{\text {min }}^{2}\right)$ & $\max \left(\eta_{\text {min }}^{2}\right)$ & $\operatorname{mean}\left(\eta_{\max }^{2}\right)$ & $\operatorname{mean}\left(\eta_{\min }^{2}\right)$ & $\max \left(\eta_{\text {min }}^{2}\right)$ & $\operatorname{mean}\left(\eta_{\max }^{2}\right)$ \\
\hline 20 & 10 & 0.0020 & 0.023 & 0.34 & 0.0020 & 0.026 & 0.35 \\
\hline 20 & 20 & 0.00099 & 0.016 & 0.77 & 0.0010 & 0.021 & 0.77 \\
\hline 50 & 10 & 0.00057 & 0.0087 & 0.11 & 0.00066 & 0.0087 & 0.11 \\
\hline 50 & 20 & 0.00029 & 0.0063 & 0.3 & 0.00035 & 0.0042 & 0.31 \\
\hline 50 & 50 & 0.00016 & 0.0021 & 0.91 & 0.00016 & 0.0025 & 0.91 \\
\hline 100 & 10 & 0.00017 & 0.0022 & 0.035 & 0.00017 & 0.0020 & 0.035 \\
\hline 100 & 20 & $9.3 \mathrm{e}-05$ & 0.0014 & 0.097 & $9.5 \mathrm{e}-05$ & 0.0014 & 0.1 \\
\hline 100 & 50 & $3.8 \mathrm{e}-05$ & 0.00046 & 0.39 & $4.2 \mathrm{e}-05$ & 0.0005 & 0.4 \\
\hline 200 & 10 & $5.2 \mathrm{e}-05$ & 0.00096 & 0.0099 & $5.5 \mathrm{e}-05$ & 0.00059 & 0.011 \\
\hline 200 & 20 & $2.3 \mathrm{e}-05$ & 0.00029 & 0.028 & $2.8 \mathrm{e}-05$ & 0.00035 & 0.031 \\
\hline 200 & 50 & $1.1 \mathrm{e}-05$ & 0.00016 & 0.11 & $1.1 \mathrm{e}-05$ & 0.00018 & 0.12 \\
\hline
\end{tabular}

Table S5: The distribution of $\eta_{\min }^{2}$ in the IND and DEP datasets. For each pair $(n, p)$, we sampled 1,000 data matrices and computed the mean and the maximum of the associated values of $\eta_{\min }^{2}$ with $\sigma_{\beta}=0.5$. The mean of $\eta_{\max }^{2}$ is listed for comparison. 


\section{S3 Numerical evidence for $\eta_{\min }^{2} \approx 0$}

Recall that in Section 2.3, when we introduced our adaptive strategy for choosing $\omega$ for ICF, we assumed that $\eta_{\min }$ is zero. Using (19) and (20), one can show that by omitting sufficiently small $\eta_{\min }^{2}$, the induced error on $\rho\left(\boldsymbol{\Psi}\left(\omega^{\star}\right)\right)$ is $c \eta_{\min }^{2}$ for some $c \in(0,1)$. Hence, it is fine to neglect $\eta_{\min }^{2}$ as long as it is less than, say 0.01 . We only need to verify this assumption for even $p$, since if $p$ is odd, $\eta_{\min }$ is always zero. It turned out that this assumption holds very generally, once we have a moderately large sample size and $p$ is not too small.

We used the two GWAS datasets described in the main text, IND and DEP, to examine how fast $\eta_{\min }^{2}$ decreases to zero. As will be shown later in the results, $\eta_{\min }^{2}$ decreases as either $n$ or $p$ increases; therefore, we only considered small values for $n$ and $p$ in this study. For each pair $(n, p)$, we randomly sampled 1,000 data matrices $\boldsymbol{X}$ from each dataset, and computed the mean and the maximum of the associated values of $\eta_{\min }^{2}$. We still used $\sigma_{\beta}=0.5$. The results shown in Table S5 indicate that it is very safe to assume $\eta_{\min }^{2} \approx 0$ when the sample size is large, say greater than 100. We did the same experiments with our simulated datasets used in Section S1 and made very similar observations. We did observe that $\eta_{\min }^{2}$ tends to be larger when the data has strong collinearity or a heavy-tailed distribution (i.e. the log-normal data). But as long as $n \geq 100$ and $p \geq 10$, it is safe to assume that $\eta_{\min }^{2}$ is negligible (less than 0.01). Table S5 also includes the mean of $\eta_{\max }^{2}$. By direct calculations, one can verify that the induced relative error on $\rho\left(\boldsymbol{\Psi}\left(\omega^{\star}\right)\right)$ is approximately $\eta_{\min }^{2} / \eta_{\max }^{2}$, which is still very small in every case we considered.

\section{S4 Summary of the MCMC algorithm of fastBVSR}

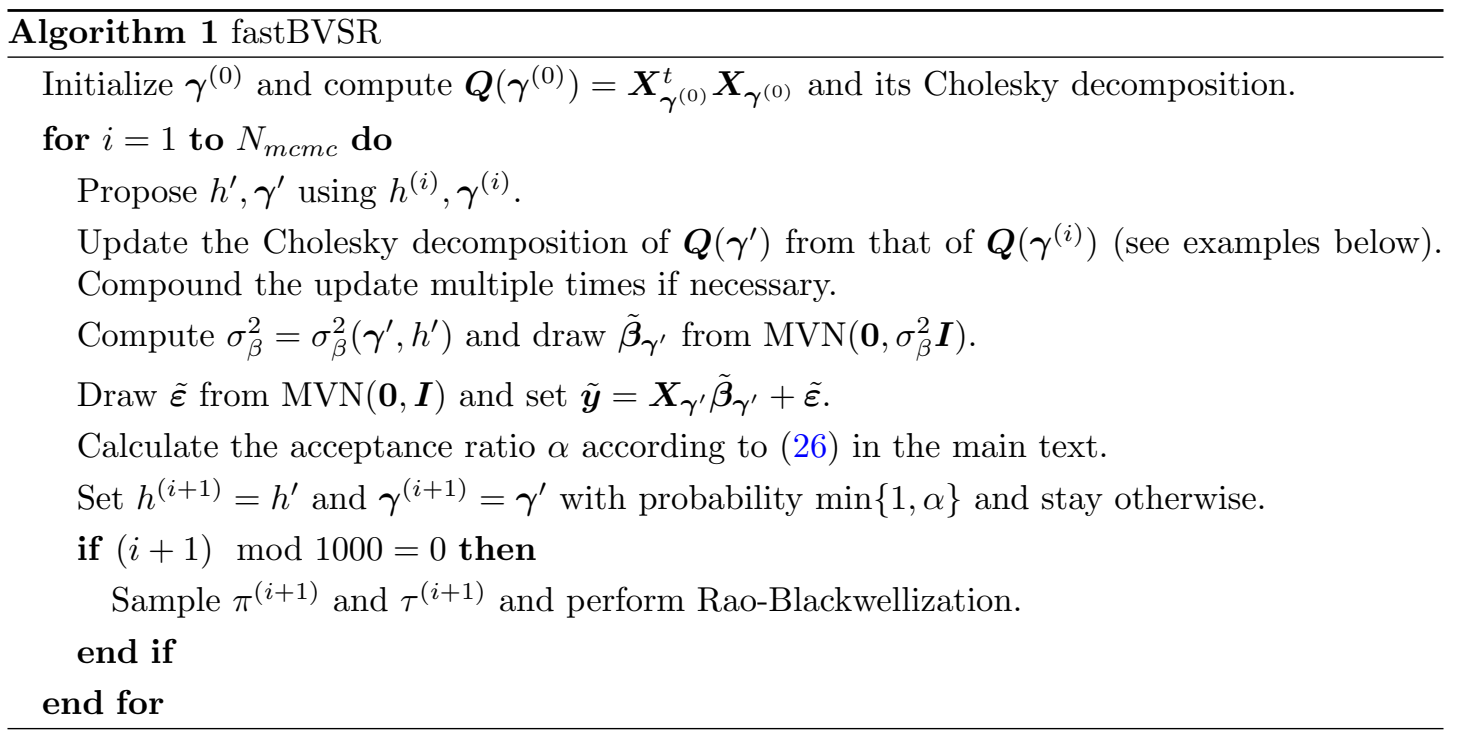

\section{S5 Examples for updating the Cholesky decomposition}

Suppose we start with

$$
\boldsymbol{X}^{t} \boldsymbol{X}=\left(\begin{array}{ccc}
9 & 3 & 6 \\
3 & 5 & 4 \\
6 & 4 & 21
\end{array}\right) \text {, and } \boldsymbol{R}=\left(\begin{array}{ccc}
3 & 1 & 2 \\
0 & 2 & 1 \\
0 & 0 & 4
\end{array}\right)
$$


such that $\boldsymbol{X}^{t} \boldsymbol{X}=\boldsymbol{R}^{t} \boldsymbol{R}$.

Add a covariate To add one covariate, we attach it to the last column of $\boldsymbol{X}$ and denote the new matrix by $\boldsymbol{X}^{\prime}$. Suppose

$$
\left(\boldsymbol{X}^{\prime}\right)^{t} \boldsymbol{X}^{\prime}=\left[\begin{array}{cccc}
9 & 3 & 6 & 3 \\
3 & 5 & 4 & 7 \\
6 & 4 & 21 & 9 \\
3 & 7 & 9 & 20
\end{array}\right]
$$

To compute the new Cholesky decomposition $\boldsymbol{R}^{\prime}$, we solve

$$
\left[\begin{array}{cccc}
3 & 0 & 0 & 0 \\
1 & 2 & 0 & 0 \\
2 & 1 & 4 & 0 \\
r_{41} & r_{42} & r_{43} & r_{44}
\end{array}\right]\left[\begin{array}{c}
r_{41} \\
r_{42} \\
r_{43} \\
r_{44}
\end{array}\right]=\left[\begin{array}{c}
3 \\
7 \\
9 \\
20
\end{array}\right]
$$

which requires only one forward substitution to get

$$
\boldsymbol{R}^{\prime}=\left[\begin{array}{llll}
3 & 1 & 2 & 1 \\
0 & 2 & 1 & 3 \\
0 & 0 & 4 & 1 \\
0 & 0 & 0 & 3
\end{array}\right]
$$

Remove a covariate Consider removing the second covariate of $\boldsymbol{X}$ and denote the new matrix by $\boldsymbol{X}^{\prime}$ to get

$$
\left(\boldsymbol{X}^{\prime}\right)^{t} \boldsymbol{X}^{\prime}=\left(\begin{array}{cc}
9 & 6 \\
6 & 21
\end{array}\right) \text {, and } \tilde{\boldsymbol{R}}=\left(\begin{array}{cc}
3 & 2 \\
0 & 1 \\
0 & 4
\end{array}\right)
$$

is obtained by removing the second column from $\boldsymbol{R}$. Note that $\tilde{\boldsymbol{R}}^{t} \tilde{\boldsymbol{R}}=\left(\boldsymbol{X}^{\prime}\right)^{t} \boldsymbol{X}^{\prime}$. To make $\tilde{r}_{32}$ zero, we introduce the Givens rotation matrix

$$
\boldsymbol{G}=\left[\begin{array}{ccc}
1 & 0 & 0 \\
0 & 1 / \sqrt{17} & 4 / \sqrt{17} \\
0 & -4 / \sqrt{17} & 1 / \sqrt{17}
\end{array}\right]
$$

Note $\boldsymbol{G}^{t} \boldsymbol{G}=\boldsymbol{I}$, and $\tilde{\boldsymbol{R}}^{t} \boldsymbol{G}^{t} \boldsymbol{G} \tilde{\boldsymbol{R}}=\tilde{\boldsymbol{R}}^{t} \tilde{\boldsymbol{R}}=\left(\boldsymbol{X}^{\prime}\right)^{t} \boldsymbol{X}^{\prime}$. The new Cholesky decomposition is then given by

$$
\boldsymbol{R}^{\prime}=\boldsymbol{G} \tilde{\boldsymbol{R}}=\left[\begin{array}{cc}
3 & 2 \\
0 & 4.123 \\
0 & 0
\end{array}\right]
$$

and the bottom row of zeros can be removed without affecting subsequent calculations.

\section{S6 Proof for the exchange algorithm}

We prove that the posterior $P(\boldsymbol{\gamma}, h \mid \boldsymbol{y})$ is the stationary distribution of the exchange algorithm by checking the detailed balance condition, i.e.

$$
\frac{K\left(\boldsymbol{\theta}^{\prime} \mid \boldsymbol{\theta}\right)}{K\left(\boldsymbol{\theta} \mid \boldsymbol{\theta}^{\prime}\right)} \frac{\int \min \left\{\alpha\left(\boldsymbol{\theta}, \boldsymbol{\theta}^{\prime}, \tilde{\boldsymbol{y}}\right), 1\right\} L\left(\tilde{\boldsymbol{y}}, \boldsymbol{\theta}^{\prime}\right) Z\left(\boldsymbol{\theta}^{\prime}\right) d \tilde{\boldsymbol{y}}}{\int \min \left\{\alpha\left(\boldsymbol{\theta}^{\prime}, \boldsymbol{\theta}, \tilde{\boldsymbol{y}}\right), 1\right\} L(\tilde{\boldsymbol{y}}, \boldsymbol{\theta}) Z(\boldsymbol{\theta}) d \tilde{\boldsymbol{y}}}=\frac{Z\left(\boldsymbol{\theta}^{\prime}\right) L\left(\boldsymbol{y}, \boldsymbol{\theta}^{\prime}\right) P\left(\boldsymbol{\theta}^{\prime}\right)}{Z(\boldsymbol{\theta}) L(\boldsymbol{y}, \boldsymbol{\theta}) P(\boldsymbol{\theta})}
$$


where $\boldsymbol{\theta}=(\boldsymbol{\gamma}, h)$ and

$$
\alpha\left(\boldsymbol{\theta}, \boldsymbol{\theta}^{\prime}, \tilde{\boldsymbol{y}}\right)=\frac{K\left(\boldsymbol{\theta} \mid \boldsymbol{\theta}^{\prime}\right)}{K\left(\boldsymbol{\theta}^{\prime} \mid \boldsymbol{\theta}\right)} \frac{L(\tilde{\boldsymbol{y}} \mid \boldsymbol{\theta})}{L\left(\tilde{\boldsymbol{y}} \mid \boldsymbol{\theta}^{\prime}\right)} \frac{L\left(\boldsymbol{y} \mid \boldsymbol{\theta}^{\prime}\right)}{L(\boldsymbol{y} \mid \boldsymbol{\theta})} \frac{P\left(\boldsymbol{\theta}^{\prime}\right)}{P(\boldsymbol{\theta})} .
$$

Let $\mathcal{Y}=\left\{\tilde{y}: \alpha\left(\boldsymbol{\theta}, \boldsymbol{\theta}^{\prime}, \tilde{\boldsymbol{y}}\right)>1\right\}$. Then,

$$
\begin{aligned}
& K\left(\boldsymbol{\theta}^{\prime} \mid \boldsymbol{\theta}\right) \int \min \left\{\alpha\left(\boldsymbol{\theta}, \boldsymbol{\theta}^{\prime}, \tilde{\boldsymbol{y}}\right), 1\right\} L\left(\tilde{\boldsymbol{y}}, \boldsymbol{\theta}^{\prime}\right) Z\left(\boldsymbol{\theta}^{\prime}\right) d \tilde{\boldsymbol{y}} \\
= & \frac{Z\left(\boldsymbol{\theta}^{\prime}\right)}{L(\boldsymbol{y}, \boldsymbol{\theta}) P(\boldsymbol{\theta})}\left[L(\boldsymbol{y}, \boldsymbol{\theta}) P(\boldsymbol{\theta}) K\left(\boldsymbol{\theta}^{\prime} \mid \boldsymbol{\theta}\right) \int_{\mathcal{Y}} L\left(\tilde{\boldsymbol{y}}, \boldsymbol{\theta}^{\prime}\right) d \tilde{\boldsymbol{y}}+L\left(\boldsymbol{y}, \boldsymbol{\theta}^{\prime}\right) P\left(\boldsymbol{\theta}^{\prime}\right) K\left(\boldsymbol{\theta} \mid \boldsymbol{\theta}^{\prime}\right) \int_{\mathcal{Y}^{c}} L(\tilde{\boldsymbol{y}}, \boldsymbol{\theta}) d \tilde{\boldsymbol{y}}\right], \\
& K\left(\boldsymbol{\theta} \mid \boldsymbol{\theta}^{\prime}\right) \int \min \left\{\alpha\left(\boldsymbol{\theta}^{\prime}, \boldsymbol{\theta}, \tilde{\boldsymbol{y}}\right), 1\right\} L(\tilde{\boldsymbol{y}}, \boldsymbol{\theta}) Z(\boldsymbol{\theta}) d \tilde{\boldsymbol{y}} \\
= & \frac{Z(\boldsymbol{\theta})}{L\left(\boldsymbol{y}, \boldsymbol{\theta}^{\prime}\right) P\left(\boldsymbol{\theta}^{\prime}\right)}\left[L(\boldsymbol{y}, \boldsymbol{\theta}) P(\boldsymbol{\theta}) K\left(\boldsymbol{\theta}^{\prime} \mid \boldsymbol{\theta}\right) \int_{\mathcal{Y}} L\left(\tilde{\boldsymbol{y}}, \boldsymbol{\theta}^{\prime}\right) d \tilde{\boldsymbol{y}}+L\left(\boldsymbol{y}, \boldsymbol{\theta}^{\prime}\right) P\left(\boldsymbol{\theta}^{\prime}\right) K\left(\boldsymbol{\theta} \mid \boldsymbol{\theta}^{\prime}\right) \int_{\mathcal{Y}^{c}} L(\tilde{\boldsymbol{y}}, \boldsymbol{\theta}) d \tilde{\boldsymbol{y}}\right] .
\end{aligned}
$$

Thus the detailed balance condition holds and the exchange algorithm leaves the posterior invariant. For a more general proof, see Andrieu and Roberts (2009).

\section{References}

Andrieu, C. and Roberts, G. O. (2009). "The pseudo-marginal approach for efficient Monte Carlo computations." The Annals of Statistics, 697-725. 9

Trefethen, L. N. and Bau III, D. (1997). Numerical linear algebra, volume 50. Siam. 3 